\title{
A Tool for Supporting the Evaluation of Active Learning Activities
}

\author{
Waraporn Jirapanthong ${ }^{(凶)}$ \\ College of Creative Design and Entertainment Technology, Dhurakij Pundit University, \\ Bangkok, Thailand \\ Waraporn.jiradpu.ac.th
}

\begin{abstract}
Active learning becomes a strategical approach for an educational principle. The student engagement become a wider concern. Many researches have been proposed to support the approach. However, one of issues is how to effectively evaluate the performance and progress of students' learning. Although, having student engagement in a classroom is vital, the evaluation of students' performance is more important. However, keeping up the details or records of students' progress is a difficult task. We therefore propose a support for instructors to evaluate the performance of their students. In particular, a prototype tool is designed and developed in order to facilitate the evaluation of activities based on an active learning class. The tool also encompasses the web service for a function of face feature recognition. Two scenarios of active learning classrooms are created in order to evaluate the prototype tool. We also plan to create a larger number of scenarios which involve different class objectives. The results show that the tool can detect and determine students with high precision values. However, the prototype tool takes a long time to be processed depending on the size and number of photos.
\end{abstract}

Keywords: Active learning $\cdot$ Education method $\cdot$ Facial recognition $\cdot$ Facial features recognition $\cdot$ Student engagement

\section{Introduction}

The concept of student engagement is becoming a concern. Active-learning techniques have emerged as strategies for instructors to promote engagement during learning classes. The engagement of students at high level increases learning and retention of the students. The engagement with students may occur outside the classroom. For in the classroom, student engagement involves the participation of students such as interaction between instructors and students or between students themselves.

The advantages of active learning are numerous: i) classroom time can be utilized in discussing higher order thinking skills rather than wasting time on lower order activities; ii) collaboration, communication and leadership skills are enhanced amongst learners; iii) students are actively engaged in a realistic learning experience.

Even though the active learning is becoming as an important approach for educational learning, it has still some challenges which include resistance from students, unprepared 
students, lack of access to technology, and heavy workload prior to and during class. Moreover, the evaluation method for the active learning approach is also concerned. Our research relies on the method and supporting tool for enhancing the active learning approach to be successful. In this paper, we present a tool for supporting instructors to evaluate the learning progress. This is to facilitate the tasks of evaluation on learners. The instructors therefore can identify participating learners and analyse the learners' performances more effectively.

In this paper, the background of active learning and facial recognition analysis is provided in Sect. 2. Our approach is presenting in Sect. 3. The experimental results, discussion and future work are following.

\section{Background}

\subsection{Active Learning}

Active learning is generally defined as any instructional method that engages students in the learning process. In short, active learning requires students to do meaningful learning activities and think about what they are doing [1]. While this definition could include traditional activities such as homework, in practice active learning refers to activities that are introduced into the classroom. The core elements of active learning are student activity and engagement in the learning process. Active learning is often contrasted to the traditional lecture where students passively receive information from the instructor.

Active learning methods that can be incorporated in the classroom: i) having multiple pauses during lectures to allow students time to reflect on what is being learnt and to consolidate their notes; ii) having brief demonstrations, or ungraded exercises, followed by a class discussion to increase student engagement; iii) incorporating small group study periods during lectures to stimulate creativity and discuss the subject matter; and iv) using case studies to involve students in doing things and thinking about what they are doing [1]. The researchers introduced engaging activities throughout traditional lectures as they stimulate learning and retention, improve students' attitudes regarding education, and enhances academic achievement [1-5]. An active learning environment notably increases student participation and collaboration with peers, where knowledge is enthusiastically shared [6]. It has further been known to stimulate creativity by promoting individual and group ideas [6]. Therefore, in a time when individuals need to be critical thinkers and problem-solvers, Active Learning provides students with the necessary tools to develop those life skills that were not necessarily on the forefront with traditional education methods. 


\subsection{Facial Recognition Analysis}

Facial recognition systems become more common. Industries and organizations are provides the services embedded with facial recognition systems.

However, some experts are still concerned regarding the algorithms, particularly when it comes to performance on faces with darker skin. Some research published by the MIT Media Lab found that some facial recognition systems performed worse when identifying an individual's gender if they were female or darker-skinned by MIT's Joy Buolamwini. Particularly, in the research, it focused on Amazon Rekognition [7] that the testing system made no mistakes when identifying the gender of lighter-skinned men. Otherwise, it mistook women for men $19 \%$ of the time and mistook darker-skinned women for men 31 percent of the time. Considering with the facial analysis software built by Microsoft, IBM, and Chinese firm Megvii, they are concerned to improve identifying similar racial and gender biases. Recently, a number of tech companies have voiced concern about the problems with facial recognition. As bias in algorithms is often the result of biased training data. However, many researches keep studying to improve the regulation and to ensure higher standards.

Although, there are little to engage with this performance, many applications and systems are still suggested to apply with the technology. A lot of researches are going on to find out the best outcome for benefits in use. For example, the gender identification test was facial analysis which spots expressions and characteristics like facial hair.

Basically, the following are the types of detection and recognition that common recognition analysis software are provided. Firstly, Labels: A label refers to any objects (e.g. flower, tree, or table), events (e.g. a wedding, graduation, or birthday party), concepts (e.g. a landscape, evening, and nature) or activities (e.g. getting out of a car). The software can detect labels in images and videos. However activities are not detected in images.

Secondly, Custom Labels: the software can identify the objects and scenes in images that are specific to some business needs by training a machine learning model. For example, a user can train a model to detect logos or detect engineering machine parts on an assembly line.

Thirdly, Faces: the software can detect faces in images and stored videos. The software shall learn where faces are detected in an image or video, facial landmarks such as the position of eyes, and detected emotions such as happy or sad. Moreover, some software have provided face searching function which is indexed into a collection of faces. The software can then be matched with faces detected in images, stored videos, and streaming video. Particularly, some software can identify the celebrities in images and stored videos. 
Fourthly, People Paths: the software can track the paths of people detected in a stored video. Some software provides path tracking, face details, and in-frame location information for people detected in a video.

Fifthly, Text Detection: the software can detect text in images and convert it into machine-readable text. This allows the analysis is further applied in other systems.

Sixthly, Unsafe Content: the software can identify images or videos for adult and with violent content.

\section{Approach}

To support the evaluation of activities during active learning, we proposed a tool to facilitate instructors to evaluate the performance and competency of learners. The prototype tool is developed. It can analyze images that are supplied as image bytes or images stored in a source folder. As shown in the figure, a part of source code allows a user to choose an image and view the estimated ages of faces that are detected in the image. The chosen image is loaded by using the displaying the image. This example shows how to unencode the loaded image bytes. The program then estimated orientation of an image and to translate bounding box coordinates. An image is loaded and its height and width are determined. The bounding box coordinates of the face for the rotated image.

The program is implemented with JavaScript and JSON. Particularly, it encompasses the Amazon web service [7] which provides a function of image recognition.

The steps to execute the program are: i) provide photos capturing the activities in the classroom in the images folder. ii) provide photos of each student in the classroom in the ref folder. iii) execute the program, entitled node; which implements the algorithm as shown in Figs. 1 and 2. iv) the program calls the web services of face recognition to identify the picture of a face. Particularly, the photos including students in various gesture are computed. When single facial features are identified, the face features are determined comparing with the individual photos. The overall configuration can be described by a vector representing the position an size of the main facial features, such as eyes and eyebrows, nose, mouth, and the shape of face outline. And v) the feature matching based on measured distances between features will be then concluded. The sets of photos for each student are created. 
function DetectFaces(imageData) \{

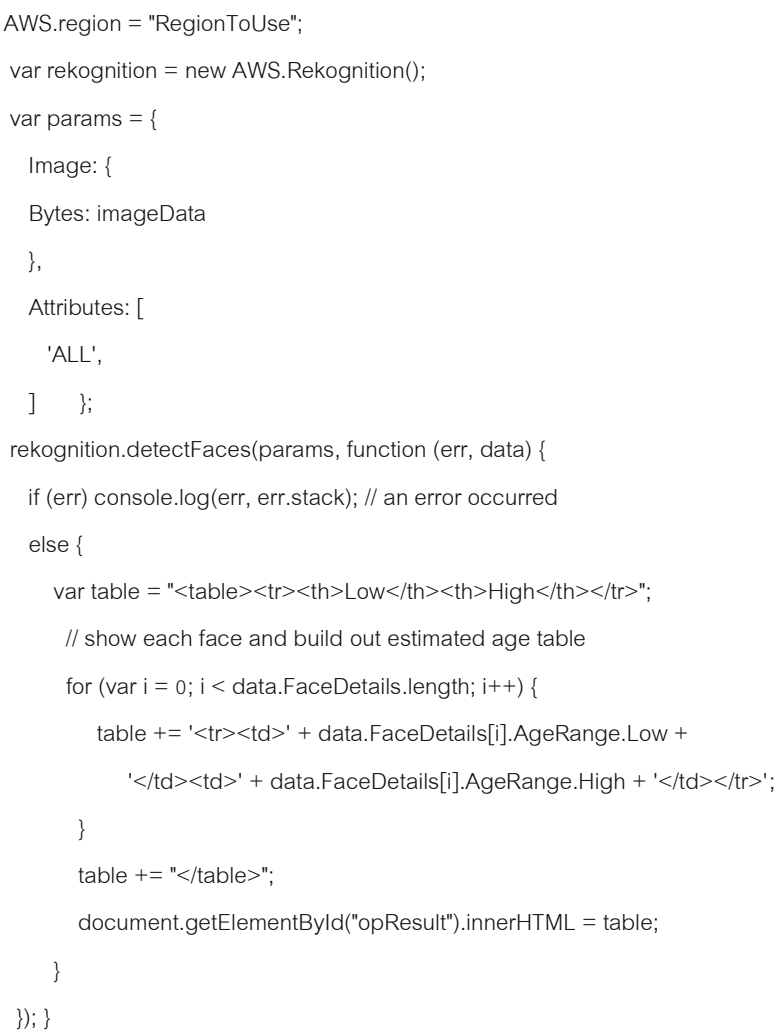

Fig. 1. A part of web service to unencode the image files. 


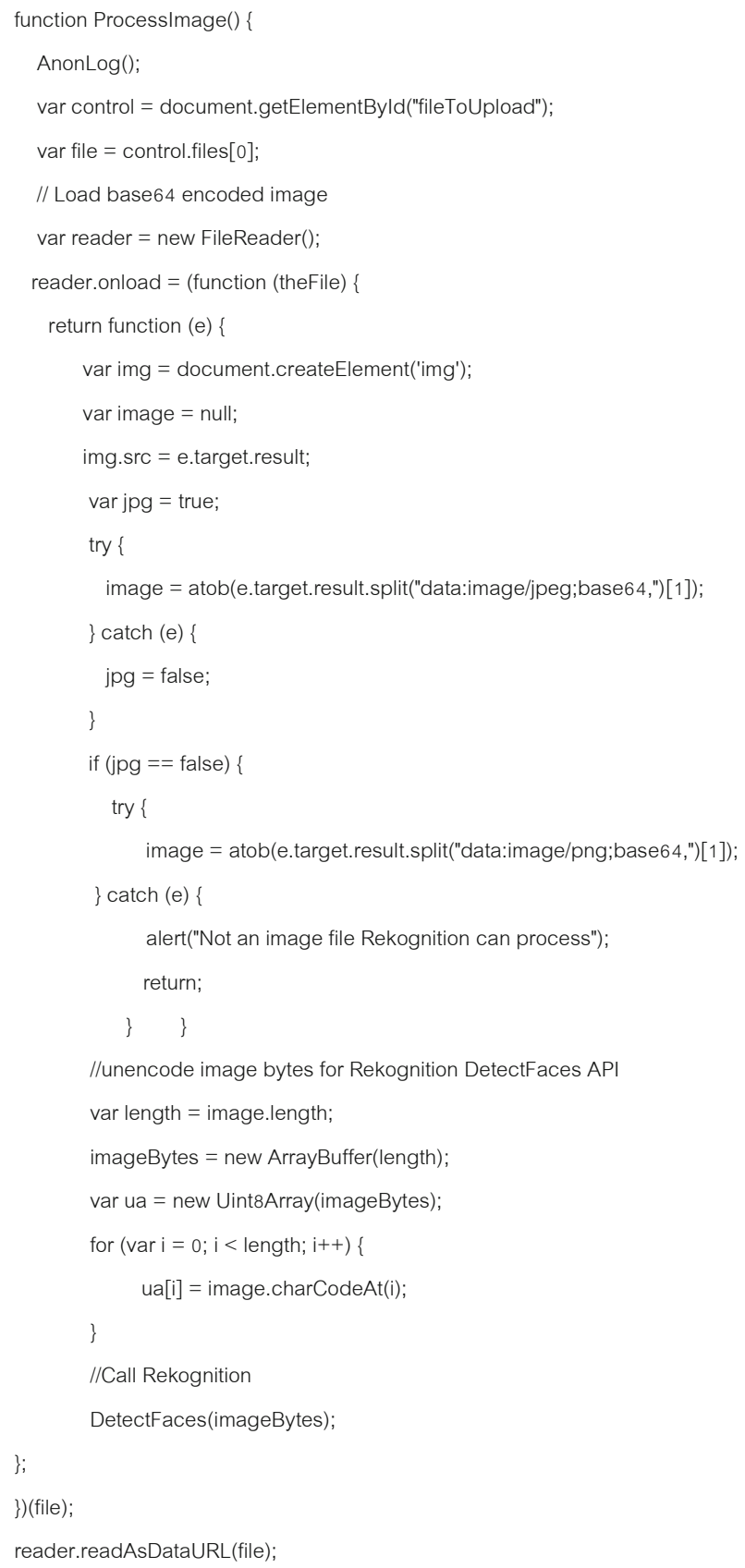

Fig. 2. A part of web service to estimated orientation of an image and to identify bounding box coordinates 


\section{Experimental Results}

To work with the program, an instructor has to capture photos during activities of active learning in a classroom. The photos then become resources for the program. As shown in Fig. 3, the photos are taken during the activities in the classroom. To evaluate the prototype, we created two scenarios of classroom. Firstly, we have a classroom with 54 students. The class is run based on active learning. Totally, the students involved the class twelve hours. The 39 photos are captured during the activities. Also, the photos of each student are provided.

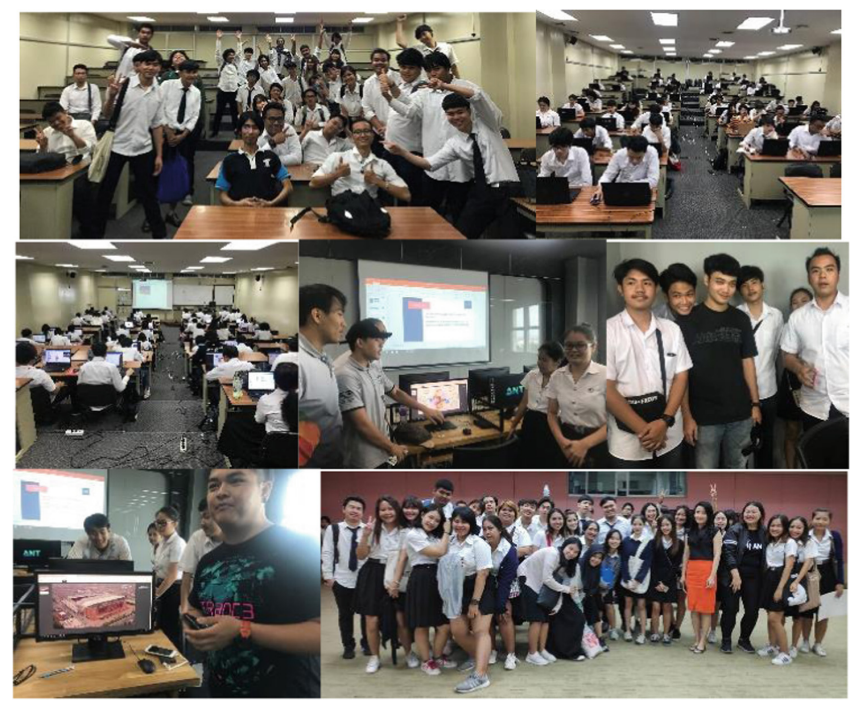

Fig. 3. Example of a set of photos capturing the activities in the classroom

Secondly, we have established a small classroom based on active learning. The class objectives are accomplished in three hours. The activities are performed according to the class. The photos are captured during the learning and provided as a resource. There are four learners participating in the classroom. Snapshots of each learner's face are provided as shown in Fig. 4. There are 9 photos taken from the class.

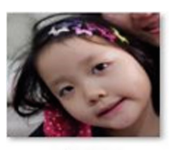

Boon

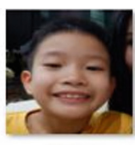

Boss

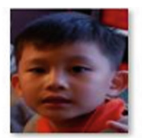

Bright

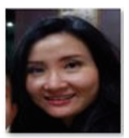

Mhee

Fig. 4. A snapshot of each learner presenting face features are provided. 
As shown in Fig. 5, the program computes by analyzing each picture. In particular, it detects the face features of each student based on reference photos. The program then create a folder of photos for each student as shown in Fig. 6.

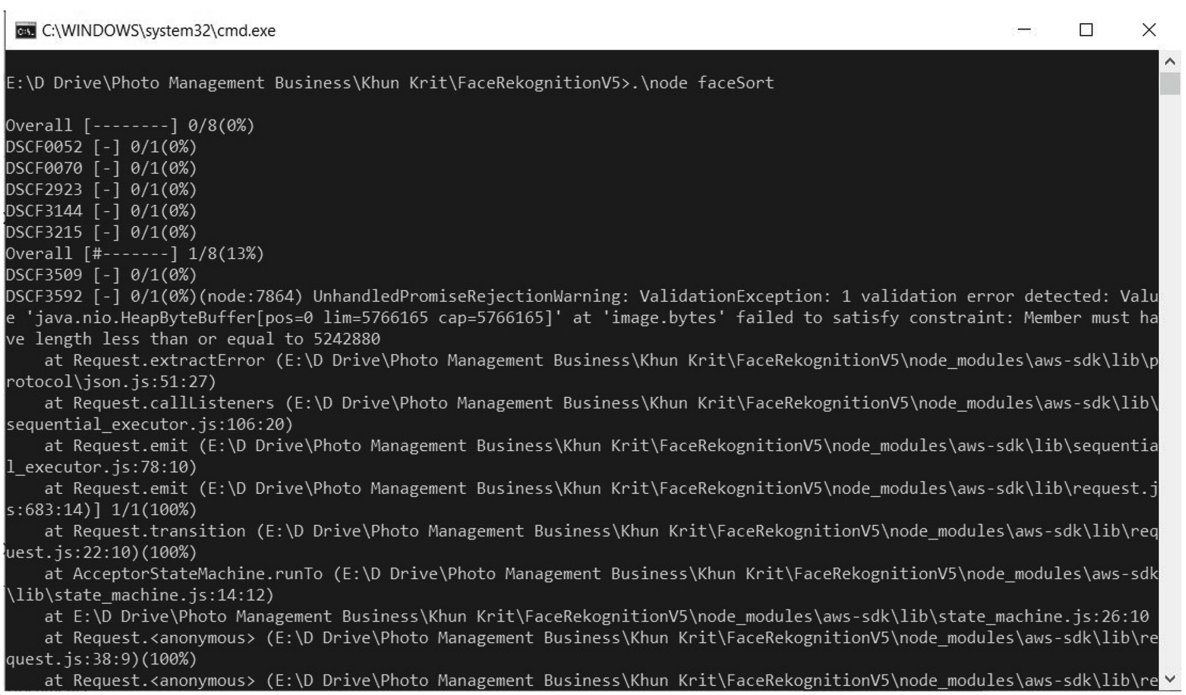

Fig. 5. All photos are computed by the program

$\begin{array}{llll}\text { Name } & \text { Date modified } & \text { Type } & \text { Size } \\ \text { III Boon } & 1 / 8 / 202010: 24 \mathrm{PM} & \text { File folder } \\ \text { II Boss } & 1 / 8 / 202010: 24 \mathrm{PM} & \text { File folder } \\ \text { Dinght } & 1 / 8 / 202010: 24 \mathrm{PM} & \text { File folder } \\ \text { Din } \text { Mhee } & 1 / 8 / 202010: 24 \mathrm{PM} & \text { File folder }\end{array}$

Fig. 6. Photos of each learner are analyzed and identified.

For the first scenario, there are 54 students with 39 taken photos. 54 folders are created by the prototype. The photos of each student are detected based on their face features. There are 51 folders identified the student correctly 100\%. Otherwise, recognition of other 3 folders are partly incorrect. One folder included 1 mismatched photo. Other two folders includes 2 mismatched photos. For the second scenario, there 4 learners with 9 photos taken during the activities. There are four folders created by the prototype. The photos of each student are detected based on their face features completely. They are totally corrected identified.

On average, the performance of our approach in terms of precision measurements in two scenarios seems to be consistently high (ranging from $94 \%$ to $100 \%$ ). 


\section{Discussion and Future Work}

Our research focuses on how to support the active learning approach. One of remaining issues is how to evaluate the performance or participating of learners effectively. Basically, recording the details of students' progress is a clumsy and tedious task. Our work here is to support the instructors to evaluate the performance of their students. The prototype tool is implemented in JavaScript in order to facilitate the evaluation of activities based on an active learning class. The tool also encompasses the Amazon web service for a function of face feature recognition. The photos of students during participating the activities in the classrooms are captured and the prototype tool then generates the folders of each student collecting only the student's photos. This assists the instructors to determine the performance and participating of each student. They then can evaluate the students' progress more effectively.

We plan to create a larger number of scenarios which involve different class objectives. It is therefore believed that the approach could be extended and enhanced to support a better way. In addition, sophisticated techniques for visualization could support the use of the prototype tool more efficiently. However, the prototype tool takes a long time to be processed depending on the size and number of photos. More work needs to be done to optimize the processing time.

\section{References}

1. Bonwell, C.C. (n.d.): Active Learning: Creating Excitement in the Classroom. https://www. ydae.purdue.edu/lct/HBCU/documents/Active_Learning_Creating_Excitement_in_the_Cla ssroom.pdf. Accessed 12 July 2014

2. Eison, J.: Using Active Learning Instructional Strategies to Create Excitement and Enhance Learning (2000). http://www.cte.cornell.edu/documents/presentations/Active\% 20Learning $\% 20 \% 20$ Creating $\% 20$ Excitement $\% 20$ in $\% 20$ the $\% 20$ Classroom $\% 20-\% 20$ Hand out.pdf. Accessed 12 July 2014

3. Michael, J.A., Modell, H.I.: Active Learning in Secondary and College Science Classrooms: A Working Model for Helping the Learner to Learn. Lawrence Erlbaum Associates, Mahwah (2003)

4. Sutherland, T.E., Bonwell, C.C. (eds.): Using Active Learning in College Classes: A Range of Options for Faculty. Jossey-Bass, San Francisco (1996)

5. Thomas, J.: The variation of memory with time for information appearing during a lecture. Stud. Adult Educ. 4, 57-62 (1972)

6. Park, E.L., Choi, B.K.: Transformation of classroom spaces: traditional versus active learning classroom in colleges. High. Educ. 68, 749-771 (2014). https://doi.org/10.1007/s10734-0149742-0

7. https://docs.aws.amazon.com/sdk-for-javascript/v2/developer-guide/getting-started-browser. html 